\title{
HARMONIC ANALYSIS OF MAXILLARY EDENTULOUS JAW SHAPES
}

\section{By}

Hamdi Mohammed, D.D.S., M.Sc.D., Ph.D., F.I.C.D.

W.M. Johnston, Ph.D., and W.J. O'Brien, Ph.D.

DOI: http://dx.doi.org/10.5915/18-3_4-12801

\section{Synopsis}

Among the factors claimed to affect the retention of full dentures is the shape of the supporting structure. Parametric evaluation through the adaption of Fourier Analysis to the surface of maxillary edentulous jaw is described in this study. It has been established that alteration in the cosine coefficient of the expanded equation affects both the right and left ridges symmetrically. Alteration of the sine coefficient affected the two sides of the ridge asymmetrically. The analysis furnished in this study will be necessary to study the relationship between jaw shape or the symmetry of such shape and the retention of full dentures.

\section{Introduction}

The forces which resist displacement of the denture in the edentulous patient are conventionally attributed to the forces of adhesion and cohesion association with the mucous membranes of the edentulous jaw, the denture base material and the separating thin film of saliva.' In attempts to analyze the forces of retention, Abdullah ${ }^{2}$ explains that the normal surface tension of the interposed saliva is chiefly responsible for the retentive forces, although O'Brien ${ }^{3}$ insists that the surface tension of liquids in isolated very thin films is substantially increased due to changes in the free energy of the material systems involved.

A previous attempt ${ }^{4}$ has been made to determine the surface area of the denture-mucosa contact interface since it is accepted on a theoretical basis that larger contact area yields proportionately larger adhesive forces..$^{2,3}$ It is also not disputed that the evaluation of the forces of denture retention is complicated by the variation of shapes taken by the edentulous jaw. It is the purpose of this study to develop a classification system for the shapes of the edentulous jaw which may be used to determine any possible correlation or lack of correlation between the shape of the edentulous jaw, the contact area, and the forces of denture retention.

\section{College of Dentistry,}

King Saud University, Riyadh 11545 Saudi Arabia and School of Dentistry, University of Michigan, Ann Arbor, Michigan 48104

\section{Mathematical Formulation and Application}

By establishing the coordinate system of the cast of the edentulous upper jaw as shown in Figure 1, the shape of a constant $\mathrm{Z}$ profile can be considered as pictured in Figure 2. The profile for any cast may be determined by measurement of coordinate points using the contour meter as described by Choudhary, Terry, Gehl, and Ryge. ${ }^{5}$ The linear width of the profile may be denoted as the period $\mathrm{P}$ of the shape to be analyzed and the shape may be represented by the following equation ${ }^{6}$ :

$$
\begin{gathered}
X=A_{0} / 2+A_{1} \cos Y^{1}+A_{2} \cos 2 Y^{1} \\
+\ldots+A_{n} \cos n Y^{1}+B_{1} \sin Y^{1}+B_{2} \sin 2 Y^{1}+\ldots \\
+\ldots B_{n} \sin n Y^{1}
\end{gathered}
$$

(Equation 1)

where:

$$
\mathrm{Y}^{1}=2 \pi / \mathrm{P}
$$

(Equation 2)

Calculation of the most accurate values of the parameters $A_{0}, A_{1}, A_{2}, \ldots, A_{n}, B_{1}, B_{2}, \ldots$, and $B_{n}$ which will reproduce the original profile shape is accomplished by the criterion of least squares curve fitting, which yields Fourier's rules ${ }^{6}$ :

$$
\begin{aligned}
& A_{0}=\frac{1}{\pi} \int \frac{Y_{0}^{1}+2 \pi x d Y^{1}}{Y_{0}} \\
& A_{j}=\frac{1}{\pi} \int \frac{Y_{0}^{1}+2}{Y_{0}^{!}}+2 \cos \mathrm{j}^{\prime} \mathrm{dY}^{\prime} \\
& B_{j}=\frac{1}{\pi} \int \frac{Y_{0}+2 \pi}{Y_{0}} X_{0} \sin \mathrm{j}^{1} \mathrm{dY}^{I_{1}}
\end{aligned}
$$

where $\mathrm{Y}_{0}^{1}$ is the corresponding $\mathrm{Y}^{1}$ value for the most negative $Y$ value and where $j^{\circ} 1,2, \ldots, n$. The value of $\mathrm{X}$ may be linearly interpolated between measured values of $\mathrm{X}$ provided that the number of measured values is equal to or greater than the number of parameters to be determined $(2 n * 2)$.

Mathematically, for any given shape which is continuous and has no undercut regions, the above analysis is perfectly accurate if $\mathrm{n}$ approaches infinity. In order to simplify the quantitative analysis of the effects of each of the parameters, a limit of $n^{6} 4$ was chosen. The above limited analysis was applied to a 
constant $\mathrm{Z}$ profile of a posterior portion of an edentulous jaw (denture area determination ${ }^{4}$ patient number $2, Z^{\mathrm{C}} 0.0 \mathrm{~mm}$.). Figure 3 shows that this limit provides an accurate representation of the shape of the profile over nearly all of the period. The numerical values of the parameters of the analysis are given in Table 1.

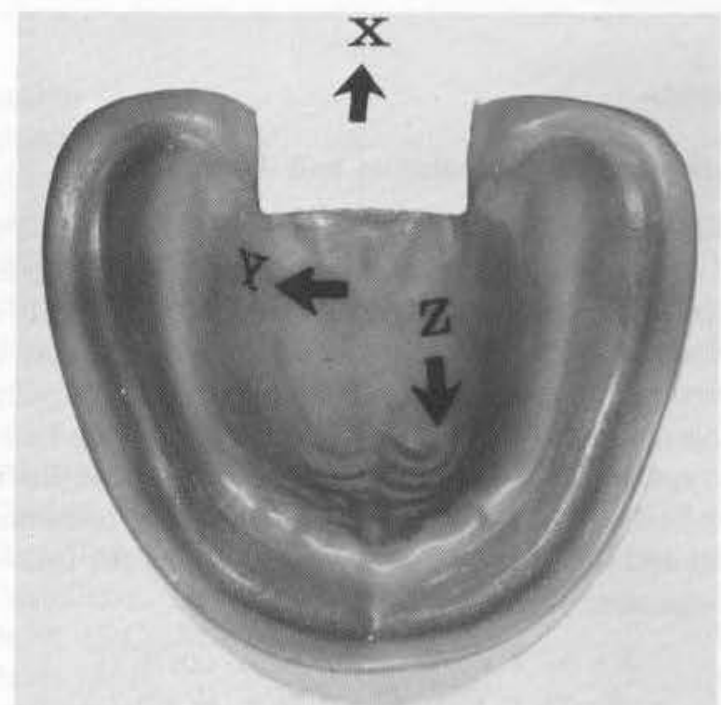

Figure 1: A cast of an edentulous upper jaw showing the orientation of the axes.

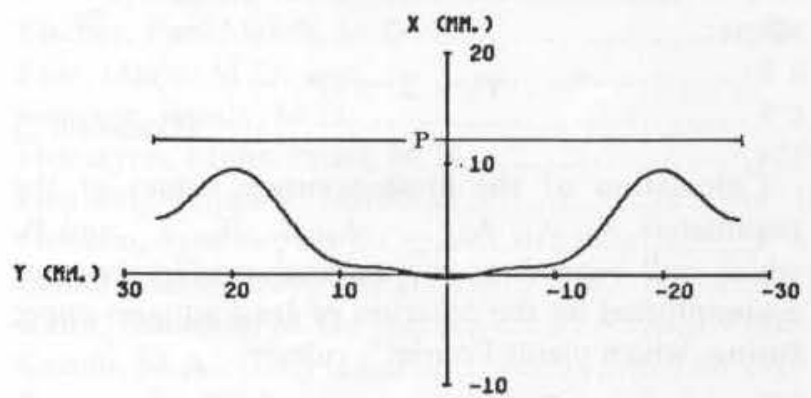

Figure 2: A constant $\mathrm{Z}$ posterior profile on an edentulous upper jaw showing the period $(\mathrm{P})$ of the shape.

\section{TABLE 1}

Values of Parameters of Fourier Analysis of Posterior Horizontal Jaw Profile shown in Figure 3.

$\begin{array}{lllll}\text { j } & \begin{array}{l}\mathrm{A}_{\mathrm{j}} \\ \text { Absolute } \\ (\mathrm{mm} .)\end{array} & \begin{array}{l}\text { Relative } \\ \text { to Period* }\end{array} & \begin{array}{l}\text { Absolute } \\ (\mathrm{mm} .)\end{array} & \begin{array}{l}\mathrm{B}_{\mathrm{j}} \\ \text { Relative } \\ \text { to Period }\end{array} \\ 0 & 7.9007 & 0.14631 & & \\ 1 & -4.4054 & -0.08158 & 0.0048 & 0.00009 \\ 2 & -0.5224 & -0.00967 & -0.0089 & -0.00016 \\ 3 & 1.7493 & 0.03239 & 0.0122 & 0.00023 \\ 4 & -1.0813 & -0.02002 & -0.0135 & -0.00025\end{array}$

*Period $(\mathrm{P})=54.0 \mathrm{~mm}$.

\section{Parametric Evaluation}

In order to consider merely the shape of the profile and in order to eliminate any considerations in the total dimensions, a practical way to evaluate a given shape of a profile using an harmonic analysis is to obtain the relative size of each parameter with respect to the period $P$. The relative values of the parameters for the representation of the profile pictured in Figure 3 are also given in Table 1. Through consideration of relative parameters, two profiles which are identical in relative shape but differ in total dimension will have different periods but identical relative shape parameters. Figure 4 shows the differences and similarities of two such profiles. The period is therefore an indicator of the total dimension of the profile.

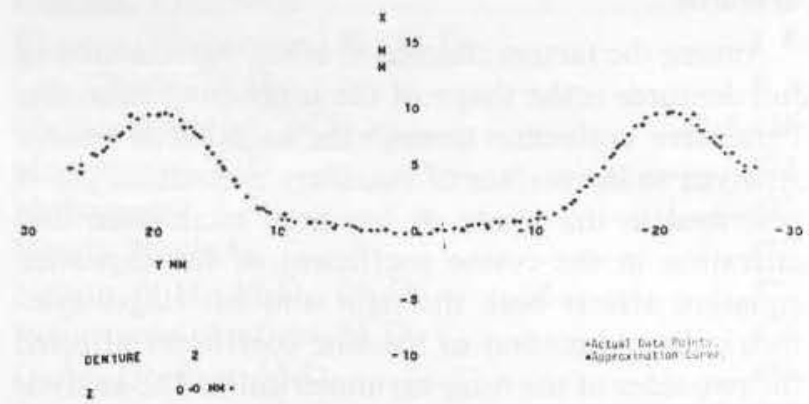

Figure 3: Fourier series representation $(n=4)$ of a constant $\mathrm{Z}$ profile. Measured profile coordinate points from the edentulous upper jaw cast using the contour meter are represented by + .
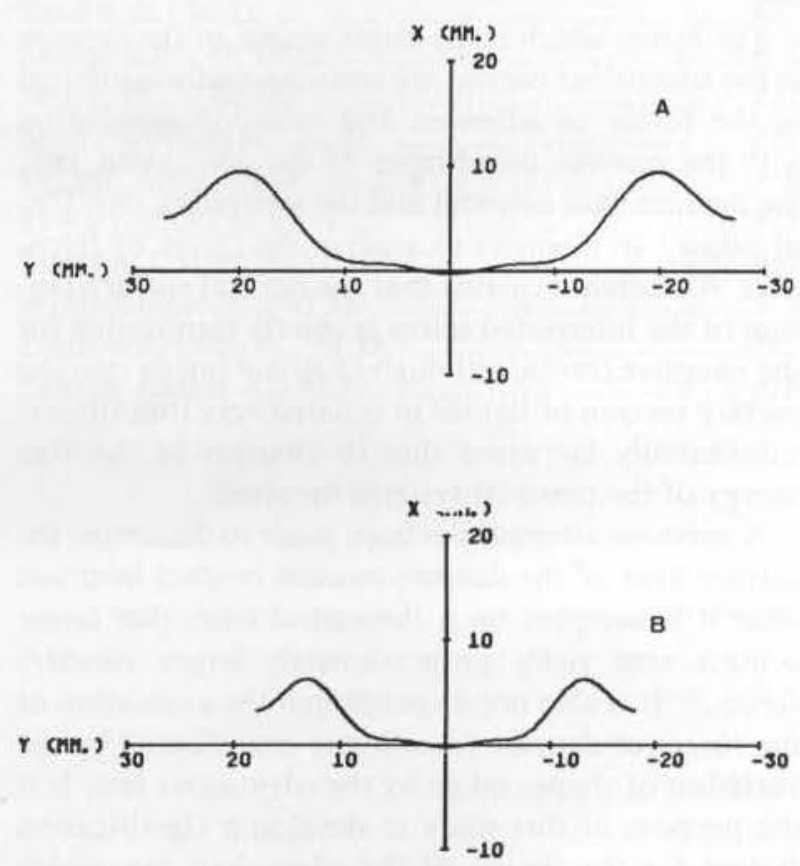

Figure 4: Two Fourier series representations with identical relative parameters but different periods. a) $\mathrm{P}=54 \mathrm{~mm}$. b) $\mathrm{P}=36 \mathrm{~mm}$.

In order to show the importance each parameter has in describing various sections of the profile, the calculated values of the parameters $\mathrm{A}_{j}$ and $\mathrm{B}_{j}$ were independently increased by some significant amount ( $2 \mathrm{~mm}$., 0.03704 relative) and each altered shape was then compared to the original representation. By inspection of Equation 3, the parameter $A_{0}$ can be seen to be twice the average value of the $\mathrm{X}$ dimen- 
sion. The value of $\mathrm{A}_{0}$ is dependent upon the choosing of the origin of the $\mathrm{X}$ axis. The effect of increasing $\mathrm{A}_{0}$ is to raise the profile uniformly as shown in Figure 5. Figure 6 shows the altered shapes plotted on the same axes as the original representation for each of the remaining $A_{j}$ parameters. Figure 7 shows this comparison for each of the $\mathrm{B}_{j}$ parameters.

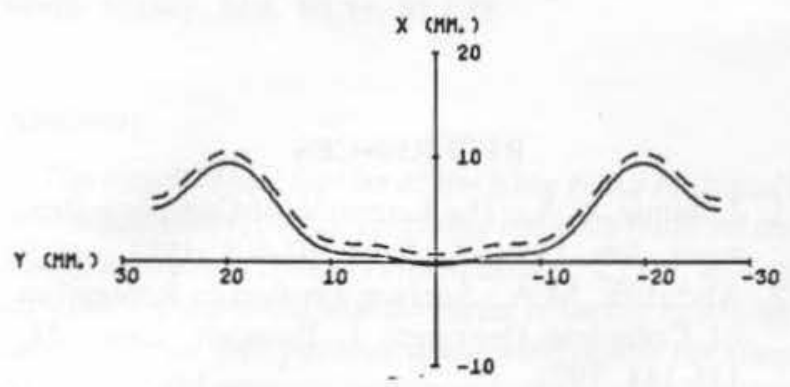

Figure 5: An altered profile and the original representation. The dotted lines show the effect of increasing $A_{0}$.
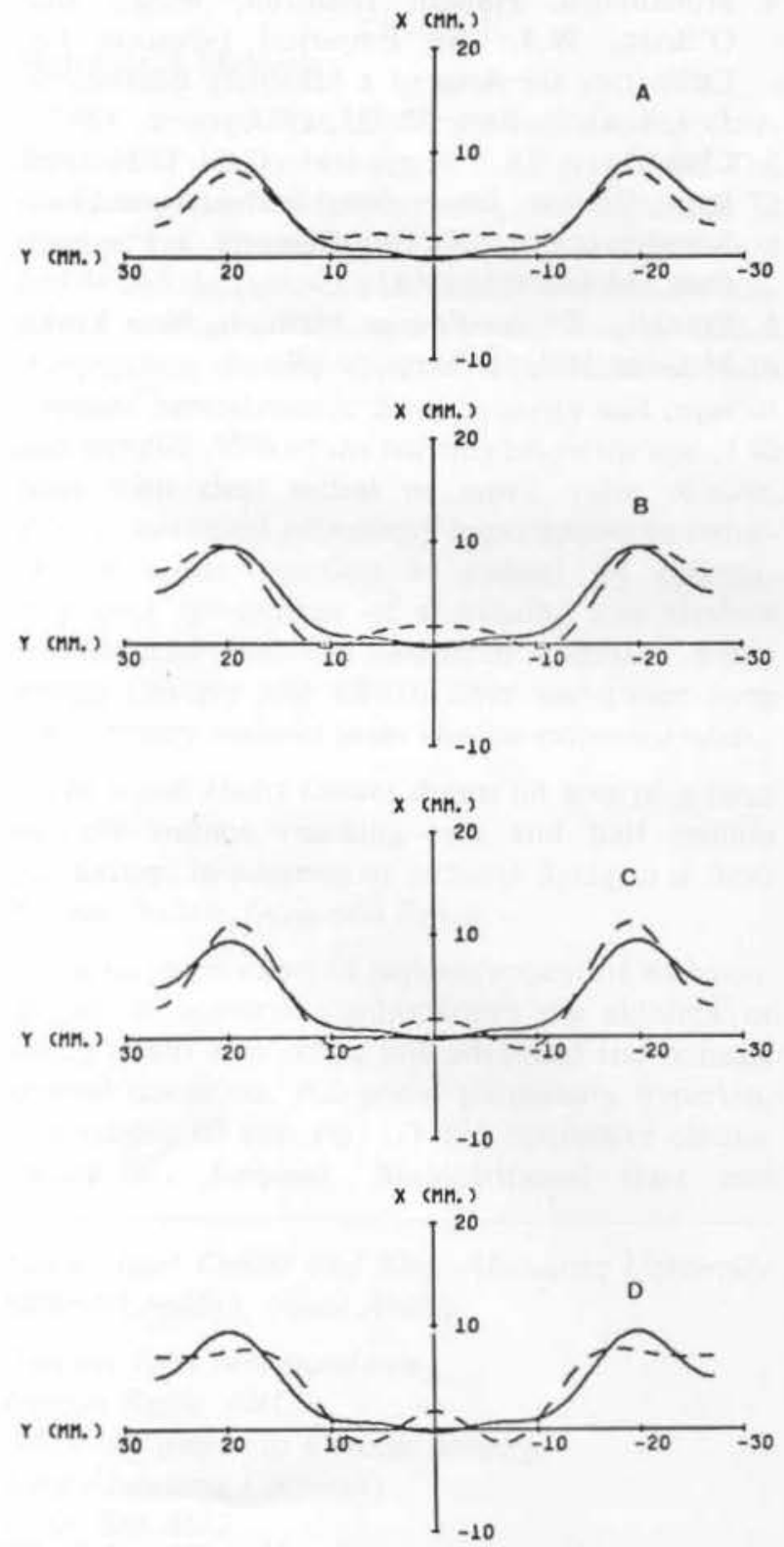

Figure 6: Altered profiles and the original represen- tation. The dotted lines show the effects of independently increasing a) $A_{1}$, b) $A_{2}$, c) $A_{3}$, and d) $A_{4}$.
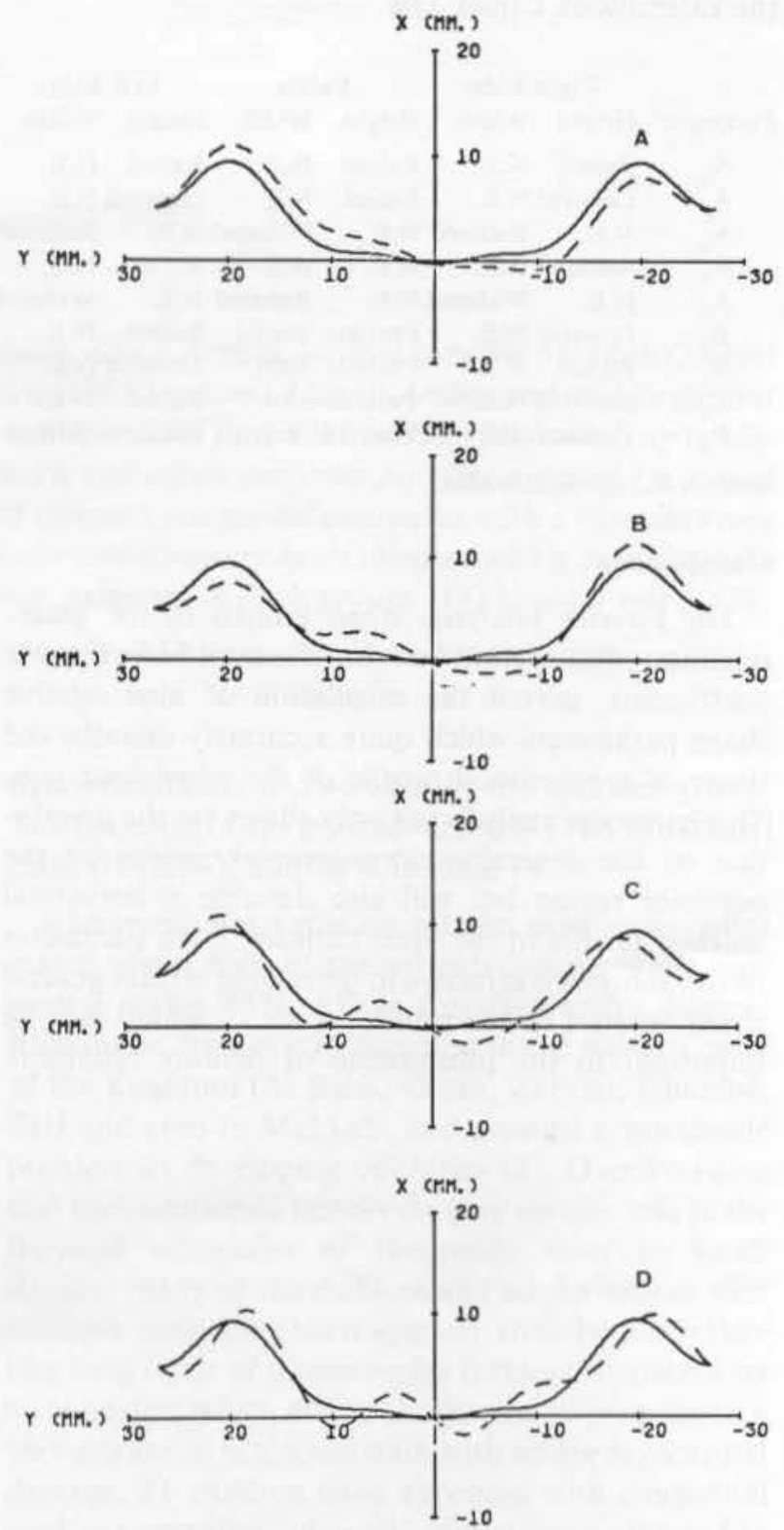

Figure 7: Altered profiles and the original representation. The dotted lines show the effects of independently increasing a) $\mathrm{B}_{1}$, b) $\mathrm{B}_{2}$, c) $\mathrm{B}_{3}$, and d) $\mathrm{B}_{4}$.

Table 2 contains a summary of the noticeable effects of independently changing each of the parameters as described above. Each of the $A_{j}$ parameters, when the harmonic analysis is applied to a constant $\mathrm{Z}$ profile of a posterior portion of an edentulous jaw, have an identical effect on both the ridges while each of the $B_{j}$ parameters have the exact opposite effect on one ridge as on the other. The $A_{j}$ parameters, the coefficients of the cosine terms in the expanded representation equation, are therefore a measure of the symmetry of the shape of the profile; the B parameters, the sine coefficients, are a measure of asymmetry. 


\section{TABLE 2}

Main Effects of Increased Fourier Analysis Parameters on Various Sections of a Posterior Profile of the Edentulous Upper Jaw.

\begin{tabular}{|c|c|c|c|c|c|c|}
\hline \multirow[b]{2}{*}{ Parameter } & \multicolumn{2}{|c|}{ Right Ridge } & \multicolumn{2}{|c|}{ Palate } & \multicolumn{2}{|c|}{ Left Ridge } \\
\hline & Height & Width & Height & Width & Height & Width \\
\hline $\mathrm{A}_{0}$ & Raised & N.E. & Raised & N.E. & Raised & N.E. \\
\hline$A_{1}$ & Lowered & N.E. & Raised & N.E. & Lowered & N.E. \\
\hline $\mathrm{A}_{2}$ & N.E. & Reduced & N.E. & Widened & N.E. & Reduced \\
\hline $\mathrm{A}_{3}$ & Raised & N.E. & N.E. & N.E. & Raised & N.E. \\
\hline $\mathrm{A}_{4}$ & N.E. & Widened & IN.E. & Reduced & N.E. & Widened \\
\hline$B_{1}$ & Lowered & N.E. & Position & Shift & Raised & N.E. \\
\hline $\mathrm{B}_{2}$ & Raised & N.E. & Position & Shift & Lowered & N.E. \\
\hline $\mathrm{B}_{3}$ & Lowered & N.E. & Position & Shift & Raised & N.E. \\
\hline$B_{4}$ & Position : & Shift & Position & Shift & Position & Shift \\
\hline
\end{tabular}

Note: N.E., no explicit effect

\section{Conclusions}

The Fourier analysis, when limited to the determination of the period and the nine most basic Fourier coefficients, permit the calculation of nine relative shape parameters which quite accurately describe the shape of a horizontal profile of the edentulous jaw. This harmonic analysis not only allows for the description of the generally more complex profile of the posterior region but will also describe a horizontal anterior profile in the same manner. Each parameter of the analysis is effective in describing certain general characteristics of the profile, some of which may be important to the phenomena of denture retention.
This analysis also permits the assessment of the symmetry of the profile.

The application of this harmonic analysis to representative horizontal profiles of the edentulous jaw may be helpful in the determination of any possible correlation between the shape of the edentulous jaw and the forces of denture retention.

\section{REFERENCES}

1. Lammie, G.A.: The Retention of Complete Dentures, Am. Dent. A. J. 55:502-508, 1957.

2. Abdullah, M.A.: Surface Tension in Rentention of Complete Dentures, J. Prosthet. Dent. 28: 141-144, 1972.

3. O'Brien, W.J.: Anomalous Behavior of Liquids in Isolated Contact with Solids, Surface Science 25:298-304, 1971.

4. Mohammed, Hamdi; Johnston, W.M.; and O'Brien, W.J.: An Empirical Equation for Estimating the Area of a Maxillary Edentulous Jaw, J. Dent. Res., 52:725, 1973.

5. Choudhary, S.C.; Terry, J.M.; Gehl, D.H.; and Ryge, Gunnar: Dimensional Stability and Fluid Sorption in Porcelain Base Dentures, J. Prosthet. Dent. 14:442-455, 1964.

6. Franklin, Philip: Fourier Methods, New York: McGraw-Hill, 1949, pp. 66-69. 